\title{
Improved bounds for the extremal number of subdivisions
}

\author{
Oliver Janzer \\ Department of Pure Mathematics and Mathematical Statistics \\ University of Cambridge \\ United Kingdom \\ oj224@cam.ac.uk
}

Submitted: Oct 24, 2018; Accepted: June 10, 2019; Published: Jul 5, 2019

(C) The author. Released under the CC BY-ND license (International 4.0).

\begin{abstract}
Let $H_{t}$ be the subdivision of $K_{t}$. Very recently, Conlon and Lee have proved that for any integer $t \geqslant 3$, there exists a constant $C$ such that $\operatorname{ex}\left(n, H_{t}\right) \leqslant C n^{3 / 2-1 / 6^{t}}$. In this paper, we prove that there exists a constant $C^{\prime} \operatorname{such}$ that $\operatorname{ex}\left(n, H_{t}\right) \leqslant$ $C^{\prime} n^{3 / 2-\frac{1}{4 t-6}}$.
\end{abstract}

Mathematics Subject Classifications: 05C35

\section{Introduction}

For a graph $H$, the extremal function $\operatorname{ex}(n, H)$ is defined to be the maximal number of edges in an $H$-free graph on $n$ vertices. This function is well understood for graphs $H$ with chromatic number at least three by the Erdös-Stone-Simonovits theorem [5, 3]. However, for bipartite graphs $H$, much less is known. For a survey on the subject, see [7]. One of the few general results, proved by Füredi [6], and reproved by Alon, Krivelevich and Sudakov [1] is the following.

Theorem 1 (Füredi, Alon-Krivelevich-Sudakov). Let $H$ be a bipartite graph such that in one of the parts all the degrees are at most $r$. Then there exists a constant $C$ such that $\operatorname{ex}(n, H) \leqslant C n^{2-1 / r}$.

Conlon and Lee [2] have conjectured that the only case when this is tight up to the implied constant is when $H$ contains a $K_{r, r}$ (it is conjectured [8] that $\operatorname{ex}\left(n, K_{r, r}\right)=$ $\left.\Omega\left(n^{2-1 / r}\right)\right)$, and that for other graphs $H$ there exists some $\delta>0$ such that $\operatorname{ex}(n, H)=$ $O\left(n^{2-1 / r-\delta}\right)$.

The subdivision of a graph $L$ is the bipartite graph with parts $V(L)$ and $E(L)$ (the vertex set and the edge set of the graph $L$, respectively) where $v \in V(L)$ is joined to 
$e \in E(L)$ if $v$ is an endpoint of $e$. In other words, it is the graph obtained by replacing every edge of $L$ by a path of length 2 .

It is easy to see that any $C_{4}$-free bipartite graph in which every vertex in one part has degree at most two is a subgraph of $H_{t}$ for some positive integer $t$, where $H_{t}$ is the subdivision of $K_{t}$. Conlon and Lee have verified their conjecture in the $r=2$ case by proving the following result.

Theorem 2 (Conlon and Lee [2, Theorem 5.1]). For any integer $t \geqslant 3$, there exists a constant $C_{t}$ such that $\operatorname{ex}\left(n, H_{t}\right) \leqslant C_{t} n^{3 / 2-1 / 6^{t}}$.

They have observed the lower bound $\operatorname{ex}\left(n, H_{t}\right) \geqslant c_{t} n^{3 / 2-\frac{t-3 / 2}{t^{2}-t-1}}$ coming from the probabilistic deletion method, and have asked for an upper bound of the form $\operatorname{ex}\left(n, H_{t}\right) \leqslant$ $C_{t} n^{3 / 2-\delta_{t}}$, where $1 / \delta_{t}$ is bounded by a polynomial in $t$. We can prove such a bound even for a linear $\delta_{t}$.

Theorem 3. For any integer $t \geqslant 3$, there exists a constant $C_{t}$ such that $\operatorname{ex}\left(n, H_{t}\right) \leqslant$ $C_{t} n^{1+\frac{t-2}{2 t-3}}=C_{t} n^{3 / 2-\frac{1}{4 t-6}}$.

It would be very interesting to know whether or not this bound is tight up to the implied constant. It certainly is tight for $t=3$ as $\operatorname{ex}\left(n, C_{6}\right)=\Theta\left(n^{4 / 3}\right)$.

We can in fact prove a slightly stronger result. For integers $s \geqslant 1$ and $t \geqslant 3$, let $L_{s, t}$ be the graph which is a $K_{s+t-1}$ with the edges of a $K_{s}$ removed. That is, the vertex set of $L_{s, t}$ is $S \cup T$ where $S \cap T=\emptyset,|S|=s$ and $|T|=t-1$, and $x y$ is an edge if and only if $x \in T$ or $y \in T$. Let $L_{s, t}^{\prime}$ be the subdivision of $L_{s, t}$.

Theorem 4. For any two integers $s \geqslant 1$ and $t \geqslant 3$, there exists a constant $C_{s, t}$ such that $\operatorname{ex}\left(n, L_{s, t}^{\prime}\right) \leqslant C_{s, t} n^{3 / 2-\frac{1}{4 t-6}}$.

This result certainly implies Theorem 3 as $L_{1, t}=K_{t}$. Moreover, we can apply Theorem 4 to obtain good bounds on the extremal number of the subdivision of the complete bipartite graph $K_{a, b}$ as well. Let us write $H_{a, b}$ for the subdivision of $K_{a, b}$. Conlon and Lee [2, Theorem 4.2] have proved that for any $2 \leqslant a \leqslant b$ there exists a constant $C$ such that $\operatorname{ex}\left(n, H_{a, b}\right) \leqslant C n^{3 / 2-\frac{1}{12 b}}$. They have also observed the lower bound $\operatorname{ex}\left(n, H_{a, b}\right)=\Omega_{a, b}\left(n^{3 / 2-\frac{a+b-3 / 2}{2 a b-1}}\right)$ (which follows from the probabilistic deletion method). Hence their upper bound is reasonably close to best possible when $a=b$, but is weak when $b$ is much larger then $a$.

Since $K_{a, b}$ is a subgraph of $L_{b, a+1}$, Theorem 4 implies the following result, by taking $s=b$ and $t=a+1$.

Corollary 5. For any two integers $2 \leqslant a \leqslant b$, there exists a constant $C_{a, b}$ such that $\operatorname{ex}\left(n, H_{a, b}\right) \leqslant C_{a, b} n^{3 / 2-\frac{1}{4 a-2}}$. 


\section{Proof of Theorem 4}

We shall use the following lemma of Conlon and Lee [2, Lemma 2.3], which is a slight modification of a result of Erdős and Simonovits [4]. Let us say that a graph $G$ is $K$-almost-regular if $\max _{v \in V(G)} \operatorname{deg}(v) \leqslant K \min _{v \in V(G)} \operatorname{deg}(v)$. Moreover, following Conlon and Lee, we say that a bipartite graph $G$ with a bipartition $A \cup B$ is balanced if $\frac{1}{2}|B| \leqslant$ $|A| \leqslant 2|B|$.

Lemma 6. For any positive constant $\alpha<1$, there exists $n_{0}$ such that if $n \geqslant n_{0}, C \geqslant 1$ and $G$ is an n-vertex graph with at least $C n^{1+\alpha}$ edges, then $G$ has a $K$-almost-regular balanced bipartite subgraph $G^{\prime}$ with $m$ vertices such that $m \geqslant n^{\frac{\alpha(1-\alpha)}{2(1+\alpha)}},\left|E\left(G^{\prime}\right)\right| \geqslant \frac{C}{10} m^{1+\alpha}$ and $K=60 \cdot 2^{1+1 / \alpha^{2}}$.

This reduces Theorem 4 to the following.

Theorem 7. For every $K \geqslant 1$, and positive integers $s \geqslant 1, t \geqslant 3$, there exists a constant $c=c(s, t, K)$ with the following property. Let $n$ be sufficiently large and let $G$ be a balanced bipartite graph with bipartition $A \cup B,|B|=n$ such that the degree of every vertex of $G$ is between $\delta$ and $K \delta$, for some $\delta \geqslant c n^{\frac{t-2}{2 t-3}}$. Then $G$ contains a copy of $L_{s, t}^{\prime}$.

Given a bipartite graph $G$ with bipartition $A \cup B$, the neighbourhood graph is the weighted graph $W_{G}$ on vertex set $A$ where the weight of the pair $u v$ is $d_{G}(u, v)=$ $\left|N_{G}(u) \cap N_{G}(v)\right|$. Here and below $N_{G}(v)$ denotes the neighbourhood of the vertex $v$ in the graph $G$. For a subset $U \subset A$, we write $W(U)$ for the total weight in $U$, ie. $W(U)=\sum_{u v \in\left(\begin{array}{l}U \\ 2\end{array}\right)} d_{G}(u, v)$.

We shall use the following simple lemma of Conlon and Lee [2, Lemma 2.4].

Lemma 8. Let $G$ be a bipartite graph with bipartition $A \cup B,|B|=n$, and minimum degree at least $\delta$ on the vertices in $A$. Then for any subset $U \subset A$ with $\delta|U| \geqslant 2 n$,

$$
\sum_{u v \in\left(\begin{array}{c}
U \\
2
\end{array}\right)} d_{G}(u, v) \geqslant \frac{\delta^{2}}{2 n}\left(\begin{array}{c}
|U| \\
2
\end{array}\right)
$$

In other words, the conclusion of Lemma 8 is that $W(U) \geqslant \frac{\delta^{2}}{2 n}\left(\begin{array}{c}|U| \\ 2\end{array}\right)$.

In the next definition, and in the rest of this paper, for a weighted graph $W$ on vertex set $A$, if $u, v \in A$, then $W(u, v)$ stands for the weight of $u v$. Moreover, we shall tacitly assume throughout the paper that $s \geqslant 1$ and $t \geqslant 3$ are fixed integers.

Definition 9. Let $W$ be a weighted graph on vertex set $A$ and let $u, v \in A$ be distinct. We say that $u v$ is a light edge if $1 \leqslant W(u, v)<\left(\begin{array}{c}s+t-1 \\ 2\end{array}\right)$ and that it is a heavy edge if $W(u, v) \geqslant\left(\begin{array}{c}s+t-1 \\ 2\end{array}\right)$.

Note that if there is a $K_{s+t-1}$ in $W_{G}$ formed by heavy edges, then clearly there is an $L_{s, t}$ in $W_{G}$ formed by heavy edges, therefore there is an $L_{s, t}^{\prime}$ in $G$.

The next lemma is one of our key observations. 
Lemma 10. Let $G$ be an $L_{s, t}^{\prime}$-free bipartite graph with bipartition $A \cup B,|B|=n$ and suppose that $W(A) \geqslant 8(s+t)^{2} n$. Then the number of light edges in $W_{G}$ is at least $\frac{W(A)}{4(s+t)^{3}}$.

Proof. Let $B=\left\{b_{1}, \ldots, b_{n}\right\}$. Let $k_{i}=\left|N_{G}\left(b_{i}\right)\right|$ and suppose that $k_{i} \geqslant 2(s+t-2)$ for some $i$. As $G$ is $L_{s, t}^{\prime}$ free, there is no $K_{s+t-1}$ in $W\left[N_{G}\left(b_{i}\right)\right]$ formed by heavy edges. Thus, by Turán's theorem, the number of light edges in $N_{G}\left(b_{i}\right)$ is at least

$$
(s+t-2)\left(\begin{array}{c}
\frac{k_{i}}{s+t-2} \\
2
\end{array}\right)=\frac{1}{2} k_{i}\left(\frac{k_{i}}{s+t-2}-1\right) \geqslant \frac{k_{i}^{2}}{4(s+t-2)} .
$$

But

$$
\sum_{i: k_{i}<2(s+t-2)}\left(\begin{array}{c}
k_{i} \\
2
\end{array}\right)<4(s+t)^{2} n \leqslant \frac{W(A)}{2},
$$

SO

$$
\sum_{i: k_{i} \geqslant 2(s+t-2)}\left(\begin{array}{c}
k_{i} \\
2
\end{array}\right) \geqslant \frac{W(A)}{2} .
$$

Since every light edge is present in at most $\left(\begin{array}{c}s+t-1 \\ 2\end{array}\right)$ of the sets $N_{G}\left(b_{i}\right)$, it follows that the total number of light edges is at least

$$
\frac{1}{\left(\begin{array}{c}
s+t-1 \\
2
\end{array}\right)} \sum_{i: k_{i} \geqslant 2(s+t-2)} \frac{k_{i}^{2}}{4(s+t-2)} \geqslant \frac{W(A)}{4(s+t)^{3}} .
$$

Corollary 11. Let $G$ be an $L_{s, t}^{\prime}$ free bipartite graph with bipartition $A \cup B,|B|=n$, and minimum degree at least $\delta$ on the vertices in $A$. Then for any subset $U \subset A$ with $|U| \geqslant \frac{8(s+t) n}{\delta}$ and $|U| \geqslant 2$, the number of light edges in $W_{G}[U]$ is at least $\frac{\delta^{2}}{8(s+t)^{3} n}\left(\begin{array}{c}|U| \\ 2\end{array}\right)$.

Proof. By Lemma 8, we have $W(U) \geqslant \frac{\delta^{2}}{2 n}\left(\begin{array}{c}|U| \\ 2\end{array}\right) \geqslant \frac{\delta^{2}}{8 n}|U|^{2} \geqslant 8(s+t)^{2} n$. Now the result follows by applying Lemma 10 to the graph $G[U \cup B]$.

We are now in a position to complete the proof of Theorem 7 .

Proof of Theorem \%. Let $c$ be specified later and suppose that $n$ is sufficiently large. Assume, for contradiction, that $G$ is $L_{s, t}^{\prime}$-free. We shall find distinct $u_{1}, \ldots, u_{t-1} \in A$ with the following properties.

(i) Each $u_{i} u_{j}$ is a light edge in $W_{G}$.

(ii) If $i, j, k$ are distinct, then $N_{G}\left(u_{i}\right) \cap N_{G}\left(u_{j}\right) \cap N_{G}\left(u_{k}\right)=\emptyset$.

(iii) For each $1 \leqslant i \leqslant t-1$, the number of $v \in A$ with the property that for every $j \leqslant i$, $u_{j} v$ is a light edge is at least $\left(\frac{\delta^{2}}{32(s+t)^{3} n}\right)^{i} \cdot|A|$. 
As $n$ is sufficiently large, we have $|A| \geqslant n / 2 \geqslant \frac{8(s+t) n}{\delta}$, therefore by Corollary 11 there are at least $\frac{\delta^{2}}{8(s+t)^{3} n}\left(\begin{array}{c}|A| \\ 2\end{array}\right)$ light edges in $A$, so we may choose $u_{1} \in A$ such that the number of light edges $u_{1} v$ is at least $\frac{\delta^{2}}{8(s+t)^{3} n}(|A|-1) \geqslant \frac{\delta^{2}}{32(s+t)^{3} n}|A|$.

Now suppose that $2 \leqslant i \leqslant t-1$, and that $u_{1}, \ldots, u_{i-1}$ have been constructed satisfying (i),(ii) and (iii). Let $U_{0}$ be the set of vertices $v \in A$ with the property that $u_{j} v$ is a light edge for every $j \leqslant i-1$. By (iii), we have $\left|U_{0}\right| \geqslant\left(\frac{\delta^{2}}{32(s+t)^{3} n}\right)^{i-1}|A|$. Now let $U$ consist of those $v \in U_{0}$ for which $N_{G}\left(u_{j}\right) \cap N_{G}\left(u_{k}\right) \cap N_{G}(v)=\emptyset$ holds for all $1 \leqslant j<k \leqslant i-1$. Since $u_{j} u_{k}$ is a light edge for any $1 \leqslant j<k \leqslant i-1$, we have that $d_{G}\left(u_{j}, u_{k}\right)<\left(\begin{array}{c}s+t-1 \\ 2\end{array}\right)$. But the degree of every $b \in B$ is at most $K \delta$, therefore the number of $v \in A$ for which $N_{G}\left(u_{j}\right) \cap N_{G}\left(u_{k}\right) \cap N_{G}(v) \neq \emptyset$ is at most $\left(\begin{array}{c}s+t-1 \\ 2\end{array}\right) K \delta$, so $\left|U_{0} \backslash U\right| \leqslant\left(\begin{array}{c}i-1 \\ 2\end{array}\right)\left(\begin{array}{c}s+t-1 \\ 2\end{array}\right) K \delta$. But note that for sufficiently large $n$, we have $\left(\frac{\delta^{2}}{32(s+t)^{3} n}\right)^{i-1}|A| \geqslant 2\left(\begin{array}{c}i-1 \\ 2\end{array}\right)\left(\begin{array}{c}s+t-1 \\ 2\end{array}\right) K \delta$ because $\delta=o\left(\left(\delta^{2} / n\right)^{t-2} n\right)$ and $\delta=o\left(\left(\delta^{2} / n\right) n\right)$. Thus,

$$
|U| \geqslant \frac{1}{2}\left|U_{0}\right| \geqslant \frac{1}{2}\left(\frac{\delta^{2}}{32(s+t)^{3} n}\right)^{i-1}|A| .
$$

But for sufficiently large $c=c(s, t, K)$, we have $\frac{1}{2}\left(\frac{\delta^{2}}{32(s+t)^{3} n}\right)^{i-1}|A| \geqslant \frac{8(s+t) n}{\delta}$. Indeed, this is obvious when $\delta^{2} \geqslant 32(s+t)^{3} n$, and otherwise, using $\delta \geqslant c n^{\frac{t-2}{2 t-3}}$, we have

$$
\frac{1}{2}\left(\frac{\delta^{2}}{32(s+t)^{3} n}\right)^{i-1}|A| \geqslant \frac{1}{2}\left(\frac{\delta^{2}}{32(s+t)^{3} n}\right)^{t-2}|A| \geqslant \frac{1}{4\left(32(s+t)^{3}\right)^{t-2}} \cdot \frac{\delta^{2 t-4}}{n^{t-3}} \geqslant \frac{8(s+t) n}{\delta}
$$

Thus, by Corollary 11, there exists some $u_{i} \in U$ with at least $\frac{\delta^{2}}{8(s+t)^{3} n}(|U|-1) \geqslant$ $\left(\frac{\delta^{2}}{32(s+t)^{3} n}\right)^{i}|A|$ light edges adjacent to it in $U$. This completes the recursive construction of the vertices $\left\{u_{j}\right\}_{1 \leqslant j \leqslant t-1}$.

By (iii) for $i=t-1$, there is a set $V \subset A$ consisting of at least $\left(\frac{\delta^{2}}{32(s+t)^{3} n}\right)^{t-1}|A|$ vertices $v$ such that for every $j \leqslant t-1, u_{j} v$ is a light edge. We shall now prove that there exist distinct $v_{1}, \ldots, v_{s} \in V$ such that $N_{G}\left(u_{i}\right) \cap N_{G}\left(u_{j}\right) \cap N_{G}\left(v_{k}\right)=\emptyset$ for all $i \neq j$, and $N_{G}\left(u_{i}\right) \cap N_{G}\left(v_{j}\right) \cap N_{G}\left(v_{k}\right)=\emptyset$ for all $j \neq k$. It is easy to see that this suffices since then there is a copy of $L_{s, t}^{\prime}$ in $G$, which is a subdivision of the copy of $L_{s, t}$ in $W_{G}$ whose vertices are $v_{1}, \ldots, v_{s}, u_{1}, \ldots, u_{t-1}$.

We shall now choose $v_{1}, \ldots, v_{s}$ one by one. Since every $u_{i} u_{j}$ is a light edge, the number of those $v \in A$ with $N_{G}\left(u_{i}\right) \cap N_{G}\left(u_{j}\right) \cap N_{G}(v) \neq \emptyset$ for some $i \neq j$ is at most $\left(\begin{array}{c}t-1 \\ 2\end{array}\right)\left(\begin{array}{c}s+t-1 \\ 2\end{array}\right) K \delta$. Moreover, given any choices for $v_{1}, \ldots, v_{k-1} \in V$, as each $u_{i} v_{j}$ is a light edge, the number of those $v \in A$ with $N_{G}\left(u_{i}\right) \cap N_{G}\left(v_{j}\right) \cap N_{G}(v) \neq \emptyset$ for some $i, j$ is at most $(t-1)(k-$ 1) $\left(\begin{array}{c}s+t-1 \\ 2\end{array}\right) K \delta$. Therefore as long as $|V|>\left(\begin{array}{c}t-1 \\ 2\end{array}\right)\left(\begin{array}{c}s+t-1 \\ 2\end{array}\right) K \delta+(t-1)(s-1)\left(\begin{array}{c}s+t-1 \\ 2\end{array}\right) K \delta$, suitable choices for $v_{1}, \ldots, v_{s}$ can be made. Since $|V| \geqslant\left(\frac{\delta^{2}}{32(s+t)^{3} n}\right)^{t-1}|A|$, this last inequality holds for large enough $c=c(s, t, K)$. 


\section{References}

[1] N. Alon, M. Krivelevich and B. Sudakov. Turán numbers of bipartite graphs and related Ramsey-type questions. Combinatorics, Probability and Computing, 12(56):477-494, 2003.

[2] D. Conlon and J. Lee. On the extremal number of subdivisions. Int. Math. Res. Not., to appear.

[3] P. Erdős and M. Simonovits. A limit theorem in graph theory. In Studia Sci. Math. Hung., 1965.

[4] P. Erdős and M. Simonovits. Some extremal problems in graph theory. In Combinatorial theory and its applications, 1969.

[5] P. Erdős and A.H. Stone. On the structure of linear graphs. Bull. Amer. Math. Soc., 52:1087-1091, 1946.

[6] Z. Füredi. On a Turán type problem of Erdős. Combinatorica, 11(1):75-79, 1991.

[7] Z. Füredi and M. Simonovits. The history of degenerate (bipartite) extremal graph problems. In Erdős Centennial, 2013.

[8] T. Kövári, V. Sós and P. Turán. On a problem of K. Zarankiewicz. In Colloquium Mathematicum, 1:50-57, 1954. 